\title{
Les phosphodiestérases des nucléotides cycliques
}

Les nucléotides cycliques, AMPc et GMPc, sont des messagers intracellulaires impliqués dans la régulation de toutes les principales fonctions biologiques. Leur effet prend fin lorsqu'ils sont hydrolysés dans la cellule par les phosphodiestérases des nucléotides cycliques. Les phosphodiestérases de mammifères ont été classées en cinq familles sur la base de leurs caractéristiques structurales et cinétiques, et de leur modulation par divers effecteurs physiologiques et pharmacologiques. Les inhibiteurs de phosphodiestérases présentent un intérêt en tant qu'outils pharmacologiques pour augmenter la teneur intracellulaire en AMPc ou en GMPc, et, par ce biais, représentent une nouvelle classe d'agents thérapeutiques potentiels dans le traitement de diverses maladies.

\section{Bernard Muller Narcisse Komas Thérèse Keravis Claire Lugnier}

es nucléotides cycliques AMPc (adénosine 3',5' monophosphate cyclique) et (iMPc (guanosine 3',5 monophosphate cyclique)ont des messagers intracellulaires impliqués dans la régulation de toutes les principales fonctions biologiques (fonctions métaboliques, contractiles et sécrétoires). L'ne élévation de la teneur en AMPc stimule la lipolyse et la glicogénolvse, augmente la force de contraction et la fréquence cardiaques, relâche les muscles lisses et module la libération de divers neurotransmetteurs et hormones. L'importance biologique du (i.MPc a été bien établie dans la transduction du stimulus visuel et la relaxation du muscle lisse.

L.es nucléotides crcliques sont impliqués dans le mécanisme d’action de nombreuses substances endogènes (hormones, neurotransmetteurs, autacoïdes) et exogènes (drogues).
Ces agents sont capables de stimuler l'activité des enzymes responsables de la srnthèse de l'A.MPc à partir d'ATP ou de celle du (;MPc à partir de (iTP (adénylate et guanylate-crclases respectivement) (figure 1). I a dégradation des nucléotides cycliques est assurée par les phosphodiestérases des nucléotides cycliques, un sistème enzymatique complexe qui représente la seule voie phrsiologique d'hydrolyse des nucléotides cycliques en nucléotides 5 correspondants (figure 1). Cinq familles de phosphodiestérases ont été distinguées chez les mammifères (Tableau I; pour revue: [1]). Certaines familles de phosphodiestérases peuvent être inhibées plus ou moins sélectivement par divers agents pharmacologiques. L'utilisation de ces inhibiteurs vise à ralentir l'hydrolyse dés nucléotides cycliques et donc à augmenter leur teneur intracellulaire, ce qui peut modifier favorable- 


\section{RÉFÉRENCES}

1. Beavo JA, Reifsnveler I)H. Primary sequence of cyclic nucleotide phosphodiesterase isommes and the design of selective inhibitors. Trends Iharmacol Soi 1990) : 11 : 15()$-5$.

2. Nicholson (:I), Challis RA, Shahid M. Differential modulation of tissue function and therapeutic potential of sclective inhibitors of cyclic nucleotide phosphodiesterase isocnzomes. Trends Pharmacol Sa 1991 ; 12: 19-27.

3. Hall IP. Isoenzome selective phosphodiesterase inhibitors: potential clinical uses. Br J C.lin Pharmacol 1993; 35: 1-7.

4. W'ang JH, Sharma RK, Mooibroek MJ. Calmodulin-stimulated cyclic mucleotide phosphodiesterases. In: Beavo JA, Houslay .11), eds. Molecular pharmacology of cell regrie lation, vol. 2: Cyclic mucleotide phosphodiesterases: structure, regulation and drug action. Chichester, UK: John W'iley and Sons, 199()$: 19-6()$

5. Bentley JK, Kadlecek A, Sherbert C:H, et al. Molecular cloming of cDNA cuncoding a " 63 "-kI)a calmodulin-stimulated phosphodiesterase from bovine brain. J Biol (ihem 1992; $267: 1867(2-82$.

6. Hidaka H, Tamaka T, ltoh H. Selective inhibitors of three forms of cyclic nucleotide phosphodiesterase. Trends Pharrnacol So 1984: 5: 237-9

7. Lugnier (', Follénius A, Gérard I), Stoclet JC. Bepridil and Ilunarizine as calmodulin inhibitors. liur / Pharmarol 1984: 98: $157-8$

8. Manganiello VC; Tanaka T, Murashima S. Ciclic (i.MP-stimulated crclic nucleotide phosphodiesterase. In : Beavo JA, Houslay .II), eds. Molecular pharmacology of cell reguilation, vol. 2: Cuclic mucleotide phosphodiesterases: structure, regulation and drug aclion. Chichester, L'K: John Wiley and Sons, 199()$: 61-86$

9. Charbomeau H, Prusti RK, I eTrong $\mathrm{H}$, of al. Identification of a non-catalytic cC $\mathrm{MP}$ binding domain consenved in both the c(i.MP-stimulated and photoreceptor cyclic mucleotide phosphodicsterases. Proc Nall Acad Sci (TSA 1990); 87: 288-92.

10. LeTrong $\mathrm{H}$, Beier $\mathrm{N}$, Somnenburg $\mathrm{W} \mathrm{K}$, ot al. Amino acid sequence of the crelic (BMP stimulated cyclic nucleotide phosphodiesterase from bovine heart. Biochemistry 199()$: 29: 10280-8$

11. Komas N, Le Bec A, Stoclet JC, Lugnier (: Cardiac c CMP-stimulated crelic nucleotide phosphodiesterases: effects of c(BMP analogues and drugs. Line / Pharracol (Mol Pharmacol Section) $1991 ; 206: 5-13$.

12. Muller B, Stoclet JC, I.ugnier C. C.vtosolic and membrane-bound crclic nucleotide phosphodiesterases from guinea-pig cardiac rentricles. Eur I Pharmacol a Mol Pharmarol Section) 1992 ; 225: 263-72. ment certaines fonctions biologiques (pour revues: $[2,3]$ ). L.es premiers inhibiteurs comnus de l'hydrolyse des nucléotides cycliques ont été les méthylxanthines (caféine, théophylline, isobutyl-méthylxanthine ou IBMX). Cet article propose une synthèse des principales informations sur la structure, la localisation, la régulation, la fonction des différentes familles de phosphodiestérases et sur les principaux domaines d'application clinique des inhibiteurs de phosphodiestérases.

\section{Les différentes familles de phosphodiestérases}

La dénomination des différentes phosphodiestérases a longtemps été basée sur leur ordre d'élution chromatographique à partir de résines échangeuses d'ions. Cependant, la multiplicité et la diversité tissulaire des enzymes (au moins 25 formes différentes de phosphodiestérases ont été identifiées cher les mammifères) et les caractéristiques différentes des supports chromatographiques utilisés par différentes équipes ont amené une grande confusion dans la dénomination des différentes enzymes. Récemment, grâce notamment à l'apport de la biologie moléculaire, Beavo et Reif snyder [1] ont proposé une classification des phosphodiestérases en cinq familles (PDE I à $V$ ) en fonction de leur séquence en acides aminés, de leurs proprié- tés cinétiques et de leur sensibilité a divers médiateurs physiologiques et agents pharmacologiques (Tableau I). Le clonage de plusieurs ADN complémentaires codant pour des phosphodiestérases appartenant aux différentes familles a confirmé l'existence de gènes multiples, apparentés mais distincts, codant pour les cing familles. Ia plupart de ces familles compremnent deux ou plusieurs sous-familles correspondant à des gènes fortement homologues mais distincts. Les identités de séquence entre les différents membres d'une même famille sont supérieures à $70 \%$. Les membres de chaque famille présentent approximativement $30 \%$ à $55 \%$ d'identité de séquence avec les membres des autres familles. Cette identité de séquence entre les différentes familles de phosphodiestérases porte essentiellement sur une région d'environ 270 acides aminés de l'extrémité C-terminale. Le site catalytique recouvre ce domaine conservé (figure 2). Les différents modulateurs de l'activité enzymatique $\left(\mathrm{C}_{\mathrm{a}}{ }^{2+} /\right.$ calmoduline, $\left.\mathrm{CMPC}\right)$ se fixent sur un domaine proche de l'extrémité N-terminale (figure 2).

\section{PDE I, \\ activées par le complexe calcium/calmoduline}

Cette famille de phosphodiestérases présente la propriété d'être activée

\begin{tabular}{|c|c|c|c|c|c|}
\hline \multicolumn{6}{|c|}{ Tableau I } \\
\hline & PDE I & PDE II & PDE III & PDEIV & PDE V \\
\hline Substrat(s) & $\begin{array}{c}\text { AMPc } \\
\text { et } \\
\text { GMPc }\end{array}$ & $\begin{array}{c}\text { AMPc } \\
\text { et } \\
\text { GMPc }\end{array}$ & $\begin{array}{c}\text { AMPc } \\
\text { et } \\
\text { GMPc }\end{array}$ & AMPc & GMPc \\
\hline Modulateur & $\begin{array}{l}\mathrm{Ca}^{2+}+ \\
\mathrm{CaM}+\end{array}$ & GMPc+ & GMPc- & & \\
\hline Inhibiteurs & $\begin{array}{c}\text { IBMX } \\
\text { nimodipine } \\
\text { vinpocétine }\end{array}$ & IBMX & $\begin{array}{l}\text { IBMX } \\
\text { milrinone } \\
\text { indolidan }\end{array}$ & $\begin{array}{c}\text { IBMX } \\
\text { rolipram } \\
\text { denbufylline }\end{array}$ & $\begin{array}{c}\text { IBMX } \\
\text { zaprinast }\end{array}$ \\
\hline
\end{tabular}

CaM: calmoduline; IBMX : isobutylméthylxanthine; +: activation; - : inhibition. 


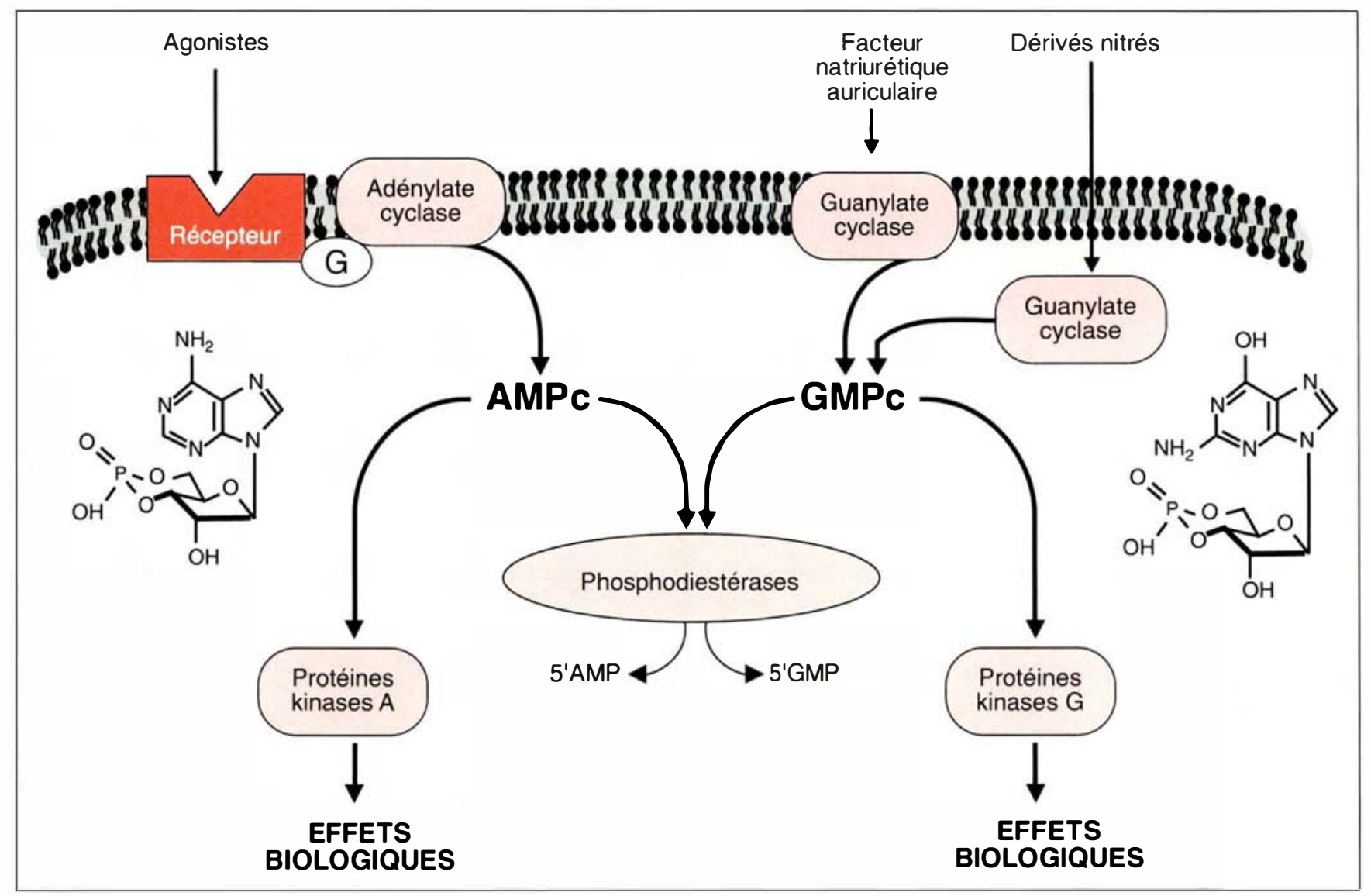

Figure 1. Représentation schématique des voies métaboliques et des effets biologiques des nucléotides cycliques. Différents agents sont capables de stimuler l'adénylate-cyclase, enzyme membranaire responsable de la synthèse d'AMPc à partir d'ATP: par exemple, les catécholamines (action sur les récepteurs $\beta$-adrénergiques ou D1-dopaminergiques), l'adénosine (action sur les récepteurs de type A2) ou la prostacycline. La synthèse de GMPc à partir de GTP est assurée par des guanylate cyclases (membranaires et cytosoliques). Le facteur natriurétique auriculaire active une guanylate cyclase membranaire, tandis qu'un facteur relaxant dérivé de l'endothélium (monoxyde d'azote) et les vasodilatateurs nitrés (nitroprussiate de sodium) stimulent l'activité d'une enzyme cytosolique. Les nucléotides cycliques exercent leurs effets biologiques par l'intermédiaire de protéine kinases et sont dégradés par les phosphodiestérases en nucléotides $5^{\prime}$ (5'AMP ou 5'GMP).

en présence dions Cä+o par une protéine thermostable de faible masse moléculaire, la calmoduline. Une PIDE I a été caractérisée pour la première fois dans le cerveau, où elle semble présente en grande quantité. Depuis, différentes sousfamilles de phosphodiestérases ont été identifiées dans divers tissus (pour revue: [4]). I.es sous-familles diffèrent essentiellement par leur répartition tissulaire et par leur propriété à utiliser l'AMPc ou le GMPc comme substrat préférentiel [1]. Les homologies de séquence primaire avec d'autres emosmes liant la calmoduline suggèrent un site de liaison $m / s \quad 11012$ vol. 9. desembre 93 pour cette protéine proche de la région N-terminale [5]. L.es antagonistes de la calmoduline (calmidazolium, flumarizine) sont capables d'inhiber l'activation par la calmoduline de nombreuses enzrmes, y compris des PIDE I [6]. La vinpocétine, en revanche, et certaines dihvdropyridines (comme la nimodipine) sont décrites comme inhibant l'activité basale des PDEI [6, 7]. Cependant, les dihudropvridines somt avant tout des inhibiteurs de l’influx calcique dans les cellules, ce qui limite à des svstèmes acellulaires leur utilisation en tant qu’inhibiteur de PIDEI. I.e role de cette famille de phosphodiestérases reste encore à définir: les agents qui angmentent le $\mathrm{Ca}^{2}{ }^{+}$intracellulaire sont susceptibles, via la calmoduline, d'activer les PIDE I et ainsi de diminuer, selon la spécificité de substrat de la PIDE I dans le tissu concerné, la teneur intracellulaire en GMPc et/ou en AMPc, (pour revue: [4]).

\section{PDE II, stimulées par le GMPc}

Initialement identifiée dans le foie, une PDE II a été mise en évidence dans de nombreus autres tissus comme la médullosurrénale, le 


\section{RÉFÉRENCES}

13. Fischmeister R, Hartell HC: Civclic A.MP phosphodiesterase and ( $\mathrm{a}^{2}{ }^{2+}$ current regulation in cardiac cells. Life Sci 1991 $48: 2365-76$.

14. Manganiello V(, Smith (J, Degerman F, Belfarge P. Corclic (i.MP-inhibited crclic nucleotide phosphodiesteratse. In: Beavo A, Houslay .11), eds. Molecular pharmacology of cell regulalion, zool. 2: Civalic mucleoticle phosphordiesterases: siruchere, regulation and doug action. (Chichester, l'k: John Wiley and Sons, 1990: 87-116.

15. Meaced F, Taira M, Moos M Jr, M al. Molecular cloning and expression of human mocoardial c(3.MP-inhibited c.t.MP phosphodiesterase. Proc . Vall Acad Sci L'S.t $1992 ; 89: 3721-5$

16. Wang I.H, Komas N, Taira .M, Manganiello V'C. Expression of human cardiac c(;.MP-inhibited (wpe III) crclic nucleotide phosphodiesterase (c(;I-PI)E) in SfY insect cells. FASEIB / 1993; 7 : 578.

17. Harrison SA, Reifsneder DH, (iallis B, Cadd $(;(;$, Bearo J.A. Isolation and characterization of bovine cardiac muscle c(i.MP inhibited phosphodiesterase : a receptor for new cardiotonic drugs. Mol Pharmacol $1986: 29: 506-14$

18. I.ugnier (, komas $x$. Iodulation of valscular cyclic nucleotide phosphodiesterat ses by crclic (i.MP: role in valsodilatation. Eur Heait / 1993 (sous presse).

19. Conti .14, Swinnen Ji: Structure and function of the rolipram sensitive, low $K_{\text {m }}$ crclic A.MP phosphodiesteratse' a lamils of highls related ensmes. In: Bearo J. Houslas 111), eds. Volecular pharmacolong of cell reguilation, vol. 2: Civclic mucleoside fihos phodiesterases: strucluere, regulalion and drug action. (Chichester, L'K: John Wiler and Sons, 1990): $243-66$.

20. I.jic (iP, kmet, P, Mchlale MII, et al Cloning and expression of (d)NA for a human low- $\mathrm{K}_{m}$, rolipran sensitive crelic A.MP phosphodiesterase. Mol (A ll Biol 1990) 10: $2678-86$

21. Muller B, L ugnier (:, Stoclet JC: Insolvement of rolipram-sensitive crclic AMP phosphodiesterase in the regulation of caldiac contractions. I Cardionesese Pharmacol $1990 \mathrm{~b}: 16: 79(\mathrm{i}-80) 3$

22. Komas N, I ugnier (:, Stoclet fo: Findothelium-dependent and independent relaxation of the rat aorta by coclic nucleor tide phosphodiesterase inhibitors. Br / Pharmacol 1991: 1(04: 495-503.

23. (illepsie P(;. Phosphodiesterases in visual transduction by rods and cones. In: Beavo JA, Houslay Mid), eds. Molecular pharmacologery of cell regualation, vol. 2 : Cyrlic mucleo tide phosphodieslerases: strurdure, regulalion and drug action. (Chichester, L'K: John Wiley and Sons, 1990: 16.3-84.

24. I.olley RN, I.ee RH. (ivclic (;.MP and photoreceptor function. lis:s:13 / 1990): 4 : coeur, l'endothélium vasculaire ou la trachée (pour revue: [8]). Un séquençage partiel d'une PDE II de glandes surrénales a pu être réalisé [9] et la séquence complète en acides aminés d'une PIDE II de coeur de boeuf a été rapportée [10]. Deux domaines différents de liaison du (iMPc ont ainsi été identifiés: ın site catalytique, pouvant fixer et hodrolvser aussi bien l'A.MPc que le (i.MPc $\left(\mathrm{K}_{\text {m }}\right.$ ou constante de Michaelis-Menten supérieure à l() $\mu \mathrm{M})$, et un site de nature allostérique, liant préférentiellement le (iMPc. Ia fixation du (B.MPc et de certains de ses analogues au site allostérique stimule l'hudrolyse de l'AMPc [11]. A l'heure actuelle, on ne comain pas d’inhibiteurs spécifiques des PIDE II. Dans les glandes surrénales, l'activité phosphodiestérase est assurée en grande partic par une PI)E II localisée presque exclusivement dans les cellules glomérullaires du cortex surrénalien. Dans ces cellules, il est bien établi, d'une part, que la tencur en (i.MPc atumente en réponse au facteur natriurétique auriculaire et, d'autre part, que le facteur nariurétique auriculaire diminue la production d'aldostérone. Ainsi, une PISE Il pourrait être impliquée dans le mécanisme d'action des agents, comme le facteur natriurétique anriculaire, connus pour angmenter la teneur intracellulaire de GiMPc et diminuer la svinthèse de stéroïdes dans les glandes surrénales [1]. Au niveau cardiatque, une PI)EII semble être présente en grande quantité au nivean membranaire [12]. Divers arguments expérimentaux laissent supposer que le mécanisme d'action des agents qui angmentent le (iMPc an niveau cardiaque impliquerait, an moins en partie, l'activation de l'hydrolyse de l'AMPc ria une PIDEll (pour revile: $[13])$.

\section{PDE III, inhibées par le GMPc}

Lne activité phosphodiestérase inhibée par le (;MPc a été identifiée initialement dans les tissus adipeux de rat. Depuis, ce trpe d'em\%'me a été purifié à partir de différents tissus comme le coeur, le muscle lisse vasculaire, la trachée on encore les pla- quettes (pour revue: [14]). I)u point de vue de leur structure, les PIDE III semblent différer des autres familles de phosphodiestérases, principalement par la présence de 44 acides aminés additionnels dans le domaine conservé (figure 2) ; cette séquence pourrait jouer un rôle important dans la régulation physiologique de ces phosphodiestérases[15]. L'analyse détaillée de la composition en acides aminés de cette famille de phosphodiestérases a permis de mettre en évidence des sites potentiels de phosphorylation et, dans l'extrémité N-terminale, une région hydrophobe susceptible d'être responsable de l'ancrage de ces enzimes dans les membranes [15, 16]. Les PIDE III hydrolysent I'AMPc et le GMPc avec des valeurs de $K_{11}$ comprises entre $(0,1 \mu \mathrm{M}$ et (), $2 \mu \mathrm{M}[15-17]$. Cependant, la vitesse d'hydrolyse de l'AMPc est dix fois plus élevée que celle du GMPc qui se comporterait comme un inhibiteur compétitif de l'hydrolyse de l'AMPc [17]. Au niveau des plaquettes et du muscle lisse vasculaire, l'inhibition de l'hydrolyse de l'AMPc par le (i.MPc pourrait expliquer laction svinergique des substances augmentant le (iMPc et des substances angmentant l'AMPc [18]. Différents agents pharmacologiques sont capables d'inhiber les PIEE III (amrinone, milrinone, enoximone, indolidan...) : ils présentent des propriétés inotropes positives, vasodilatatrices et antiagrégantes plaquettaires (pour revues: [2, 3, 14]).

\section{PDEIV, hydrolysant spécifiquement I'AMPc}

L'ne PIDE IV a été identifiée dans de très nombreux tissus comme le cerveau, le coeur, l'endothélium vasculaire, le muscle lisse vasculaire et la trachée (pour revue: [19]). Au moins cinq enzymes différentes ont été clonées; celle des monocytes humains présente $ı \mathrm{~K}_{\mathrm{m}}$ vis-à-vis de l'AMPc de $3 \mu \mathrm{M}[2(0)$. Cette famille de phosphodiestérases est inhibée spécifiquement par le composé Ro 2()-1724, le rolipram, et la denbufylline.

Les PIDE Ill et IV sont souvent décrites comme hydrolysant sélectivement I'AMPc: l'analyse du rôle respectif 
de ces deux familles d'emzymes dans les tissus où elles coexistent (coeur, muscle lisse vasculaire et bronchique) suggère que la PDE IV serait impliquée dans la régulation de la teneur en AMPc uniquement si celle-ci a été élevée au préalable ; la PIDEIII, en revanche, serait impliquée dans la régulation de la teneur basale en AMPC [21, 22] ; cette différence pourrait s'expliquer par la différence de $K_{m}$ de ces deux enzymes vis-à-vis de l'AMPc [12].

\section{PDE $V$, spécifiques du GMPC}

Cette famille de phosphodiestérases est composée de deux sous-familles, à forte sélectivité pour le GMPc comme substrat et présentant, de plus, un site de liaison pour le GMPc. I.es séquences primaires des
PDE II et des PDE V compremnent une région à forte homologie qui pourrait contenir leur site de liaison pour le GMPc [9].

Une sous-famille de PIDE $V$ est localisée dans les cellules rétiniennes des vertébrés (pour revue: [23]). En l'absence de lumière, l'activité de cette phosphodiestérase est faible: elle peut être très rapidement stimulée par la cascade de réactions résultant de l'absorption de lumière par les photorécepteurs rétiniens (rhodopsine). La chute de la teneur en GMPc permet la fermeture d'um canal ionique et l'hyperpolarisation de la cellule (pour revue: [24]). Une autre sous-famille de PIEV V, aux propriétés enzymatiques différentes, est présente au niveau des plaquettes, du poumon et du muscle lisse vasculaire [25, 26]. Son $\mathrm{K}$ vis-di-vis du (;MPc est de l'ordre de

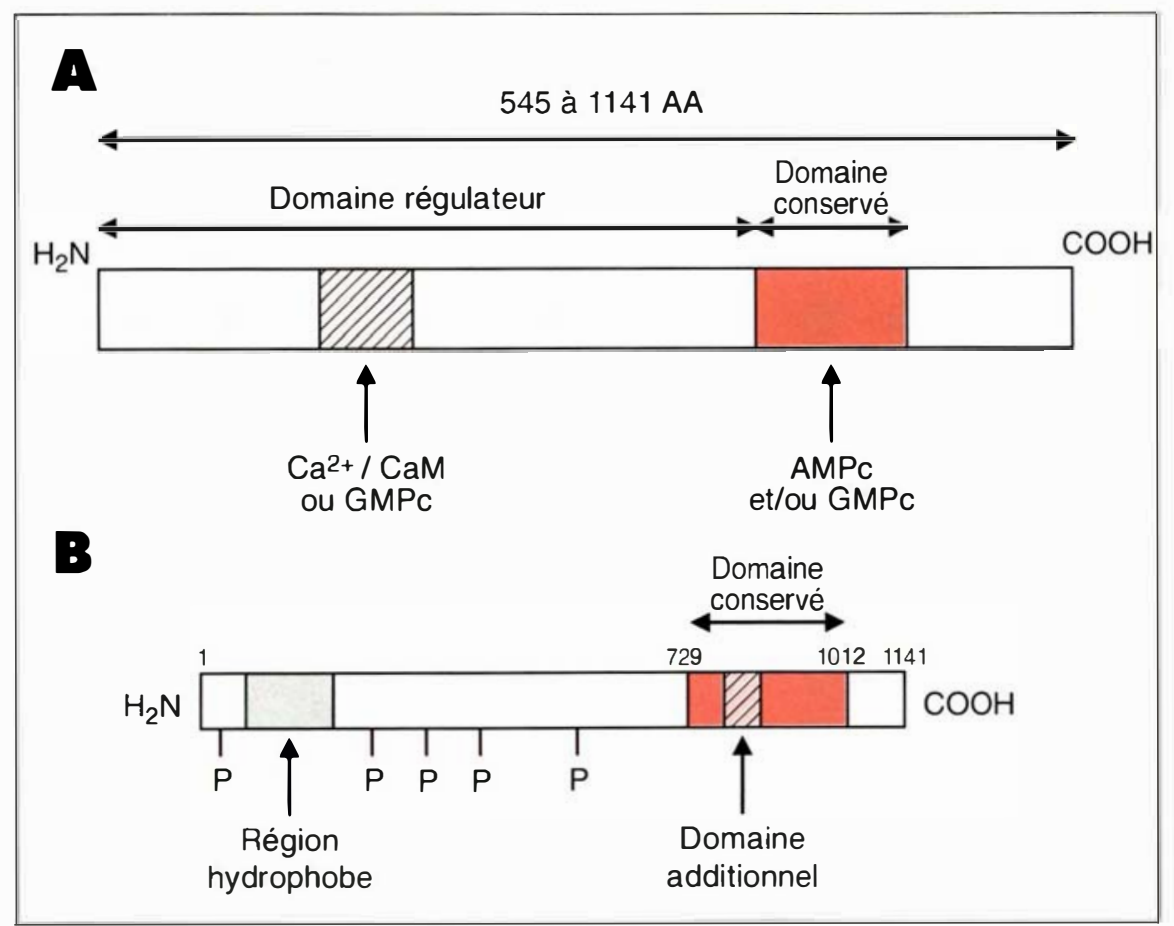

Figure 2. (A) Représentation schématique de la structure générale des phosphodiestérases des nucléotides cycliques. Dans l'extrémité Cterminale, une région de 270 acides aminés, conservée dans les différentes familles, est incluse dans le site catalytique (où le(s) substrat(s) est (sont) hydrolysé(s)). Les différents modulateurs (complexe $\mathrm{Ca}^{2+} / \mathrm{calmoduline,}$ $G M P C)$ se fixent sur un site régulateur plus proche de l'extrémité $\mathrm{N}$-terminale. (B) Représentation schématique détaillée de la structure de la PDE III clonée à partir de coeur humain $[15,16]$. Cette PDE diffère des autres PDE par la présence de 44 acides aminés additionnels dans le domaine conservé. Dans l'extrémité $\mathrm{N}$-terminale, des domaines hydrophobes ont été identifiés. Les sites potentiels de phosphorylation par la protéine-kinase activée par l'AMPC sont indiqués $(P)$.
(),3 $3 \mathrm{M}$. Elle possède également un site de liaison pour le GMPc distinct du site catalytique. L'importance éventuelle de la fixation du (:MPc sur ce deuxième site reste encore à définir. Cette sous-famille de PIDE V est inhibée spécifiquement par le zaprinast [26]. Elle pourrait jouer un rôle important dans la régulation de la relaxation des muscles lisses.

\section{Régulation des phosphodiestérases}

Dans de nombreux types cellulaires, les phosphodiestérases sont susceptibles d'être modulées, soit par phosphorylation (différents types de protéine kinases sont impliqués), soit par effet direct d'un second messager (pour revue: [27]). Ainsi, les agents qui augmentent le $\mathrm{Ca}^{2+}$ intracellulaire sont capables d'activer les PIDE I (via la calmoduline). Les agents qui élèvent la teneur en G.MPc sont, quant à eux, susceptibles à la fois d'activer une PIDE II et d'inhiber une PIDEIII. Récemment, un nouveau mode de régulation a été décrit: le mécanisme d'action du glucagon au niveau cardiaque impliquerait l'inhibition d'une PIDE III par l'intermédiaire d'une protéine de couplage liant les nucléotides guanyliques [28].

I a modulation peut aussi impliquer des cycles de phosphorylationdéphosphorylation. I a phosphorylation in vitro d'une PIDE I par les protéine kinases activées par l'AMPc ou par des protéine kinases activées par le complexe (ata+/calmoduline entraine une diminution de l'affinité des PDEl pour le complexe Ca ${ }^{2+} /$ calmoduline et une diminution de l'activation de la phosphodiestérase [29]. Cependant, il n'y a pas d'études impliquant ce phénomène à la suite de la stimulation cellulaire par un agoniste. L'activité des PIDE III peut être modulée par les agents qui augmentent la teneur intracellulaire en AMPc. Ainsi dans le foie, la stimulation par le glucagon induit la phosphorylation et l'activation d'une PIDE III par les protéine kinases activées par l'AMPc. Un effet identique a été rapporté au niveau des plaquettes à la suite de l'action de la prostacycline [3()]. L'importance phy'siologique de ces 


\section{RÉFÉRENCES}

2:). Francis $\mathrm{SH}$, Thomas MK, (orbin fl) Coclic (i.llP-binding crclic (i.MP-specific phosphodiesterase from lung. In: Bearo A, Houslay .MI), eds. Molecular pharmacer loge of cell regulation, vol. 2: Cyclic mucleotide phosphodiesterases: struclure, regulation and drug action. (:hichester, L'K: John Wiler and Sons, 199(): $117-40$.

26. I.ugnier (: Schoeffier P, I.e Bec A, Surouthou F., Stoclet J(:. Selective inhibition of crclic mucleotide phosphodiesterase of human, bovine and rat anta. Biochem Phar macol 1986; 3.5: 1743-51

27. (omti .M, Jin SI.C, Monaco I., Repaske DR, Swinnen JV: Hormonal regulation of crclic nucleotide phosphodiesterases. Endon Rev 1991: 12: 218-54.

פ8. Brechler V', Paroine (', Hanl R, (jarbar\% F, Fischmcister R, Pecker F. Inhibition by glucagon of the c(;.MP-inhibited low-K'n c.A.MP phosphodiesterase in heart is mediated by a pertussis toxin-sensitive (-

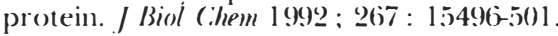
29. Sharma RK, Wang Jll. Differential regulation of bovine brain calmodulindependent crclic nucleotide phosphodiesterase isoenzimes by cyclic AMP dependent protein kinase and calmodulin-dependent protein phosphatase. Hor . Vall Arad Soi L'SA 1985 ; 82: $26(0) 3-7$.

30. Macphee (:I, Reifsuyder DH, Moore TA, I.erea K.M, Beavo IA. Phosphomylation results in actiation of a (AMP phosphodiesterase in human platelets. I Biol Chem 1987: 26(3:3: 10.3:3.3-8

31. Machtel H. Potential antidepressant activity of rolipram and other selective crclic adenosine $3,55^{\circ}$-monophosphate phosphodiesterase inhibitors. Neuropharmacology 1983: 22): $267-72$.

32. Hebenstreit GF, Fellerer $\mathrm{K}$, Fichte $\mathrm{k}$, el al. Rolipram in major depressive disorder : results of a double blind comparative study with imipramine. Pharmacopsychialry 1989 ; 22) : 15(2-60).

33. Fischer TA, Erbel R, Treese N. current status of phosphodiesterase inhibitors in the treatment of congestive heart failure. Drugs 1992); 44 : 920-45.

34. Muller B, I.ugnier (:, Stoclet JC:. Impli(ation of crelic A.MP in the positive inotropic effects of cyclic (;.MP-inhibited cyclic A.MP phosphodiesterase inhibitors on guinea pig isolated left atria. / Corrlioness Pharmarol 19)(): 15): 444-i)l.

35. Komas $x$, lugnice (i, I.e Bec A, Serradeil-I ce (;al C;, Barthéléms (; Stoclet IC. Differential sensitivit to cardiotonic drugs of cyclic A.MP phosphodiesterases isolated from canine ventricular and sincatrial-enriched tissues. / (iardionossc Pharmacol 1989): 14: 2013-20)

36. Mood MA, Hess Ml. Review: I.ongterm oral therapy of congestive heart failure with phosphodiesterase inhibitors. Am I Med Sci 1989): 297\%: 10:5-13.

37. Torphy TJ, Lndem BJ. Phosphodiesterase inhibitors: new opportunities for the treatment of asthma. Thorax 1991: 46: mécanismes de régulation reste encore à établir. D'une manière générale, on peut considérer que ce mode de régulation sert de rétrocontrôle négatif au sv'stème, permettant de diminuer plus rapidement la teneur intracellulaire en nucléotides crcliques. Dans les adipocrtes, la PIDE III est sujette à une double régulation : d'une part, la stimulation $\beta$-adrénergique induit l'activation de l'enzyme (par phosphorylation via les protéine kinases activées par l'AMPc) ; d'autre part, l'insuline peut également activer cette phosphodiestérase de manière indirecte par un mécanisme faisant intervenir une cascade de réactions de phosphorylation (intervention probable d'une tyrosine kinase puis d'une sérinekinase) [14]. Les facteurs de croissance, tout comme l'insuline, agissent sur des récepteurs à activité tyrosine-kinase ; certains facteurs de croissance sont commus pour stimuler l'hydrolyse de l'AMPc. Cependant, le lien entre l'activation de la PIDE III et les effets biologiques de l'insuline et des facteurs de croissance reste encore à établir. Les études du mode de régulation des différentes phosphodiestérases sont en plein développement à l'heure actuelle.

\section{Domaines d'application clinique des inhibiteurs de phosphodiestérases}

L'intérêt suscité par les inhibiteurs de phosphodiestérase en tant qu'agents thérapeutiques repose sur l'existence de différentes familles de phosphodiestérases, leur sensibilité différente pour certains inhibiteurs et leur répartition tissulaire différente. Ainsi, il est possible d'augmenter sélectivement la teneur d'un nucléotide cyclique domné dans un type cellulaire domné afin de modifier une fonction précise. Ainsi, les inhibiteurs sélectifs de certaines familles de phosphodiestérases présentent un intérêt comme agents thérapeutiques dans le traitement de diverses maladies (pour revues: [2, 3]).

\section{Dépression}

I.e rolipram, un inhibiteur de PIEE IV, est efficace sur différents modèles de dépression, y compris chez l'homme chez lequel son efficacité est comparable à celle des antidépresseurs tricýcliques [31, 32]. Un des mécanismes d'action du rolipram semble faire intervenir une augmentation de la libération de noradrénaline à partir des terminaisons nerveuses sympathiques [32].

\section{Insuffisance cardiaque}

I.e domaine principal d'application thérapeutique des inhibiteurs de phosphodiestérases a été pendant longtemps le traitement de l'insuffisance cardiaque congestive (pour revue récente: [33]). Des études in vitro et in vivo ont montré en effet que les inhibiteurs de PIDE III (amrinone, milrinone, enoximone, piroximone...) ont un effet vasorelaxant et augmentent la force de contraction cardiaque, sans provoquer d'augmentation importante du rythme cardiaque. De nombreux arguments expérimentaux sont en faveur de l'implication de l'inhibition de la PIDE III et du rôle de l'AMPc dans les effets cardiotoniques de ces substances [34]. L'ne différence de sensibilité des inhibiteurs vis-à-ris de la PIDE III entre le tissu ventriculaire et le noeud sinusal (siège du "pacemaker") pourrait expliquer la dissociation entre les propriétés inotropes positives et chronotropes positives de certains de ces agents [35]. A court terme chez l'insuffisant cardiaque, les inhibiteurs de PDE III permettent d'augmenter le débit cardiaque. Cependant, il n'existe que peu d'arguments concernant l'efficacité de la thérapie à long terme, qui ne semble pas apporter de bénéfice comparée aux traitements usuels et qui, par ailleurs, pourrait contribuer à augmenter la mortalité. L'usage des inhibiteurs de PIDE III reste pour l'instant limité au traitement à court terme de l'insuffisance cardiaque congestive aiguë (pour revue: [36]).

\section{Asthme}

A l'heure actuelle, les inhibiteurs de PDE IV sont étudiés comme agents anti-inflammatoires pour le traitement de l'asthme bronchique. Au niveau pulmonaire, les cellules 
impliquées dans la libération de médiateurs inflammatoires (mastocytes, macrophages, neutrophiles, éosinophiles, basophiles) contiennent une proportion importante de PDE IV. Les inhibiteurs de PDE IV peuvent diminuer la libération de ces médiateurs ; ils possèdent également des effets relaxants sur le muscle lisse des bronches et de la trachée [37]. Ces agents pourraient donc être particulièrement intéressants dans cet axe thérapeutique du fait de leur propriété bronchodilatatrice et anti-inflammatoire.

\section{Conclusions}

\section{et perspectives}

Les nucléotides cycliques et leur voie de dégradation par une activité phosphodiestérase ont été découverts il y a maintenant plus de 30 ans. Les dix dernières années ont révélé la complexité des phosphodiestérases. L'évolution des connaissances dans ce domaine résulte de la combinaison de différentes approchesbiochimiques, pharmacologiques et de biologie moléculaire. La biologie moléculaire devrait permettre d'apporter des informations plus précises sur les distributions tissulaires et cellulaires de chaque phosphodiestérase et sur leur mode de régulation. On peut envisager que le clonage des ADN complémentaires des différentes phosphodiestérases et leur expression dans des lignées cellulaires adéquates puissent permettre, d'une part, d'étudier les relations structurefonction des différentes enzymes et, d'autre part, de développer des inhibiteurs de plus en plus sélectifs d'une enzyme d'un type cellulaire donné. Ces études permettront non seulement de mieux comprendre le rôle joué par une enzyme précise dans la régulation d'une fonction cellulaire donnée, mais aussi d'envisager de modifier spécifiquement une fonction particulière dans une perspective thérapeutique

\section{Remerciements}

Les auteurs tiennent à remercier vivement le Pr Jean-Claude Stoclet et Mlle Viviane Martin pour la lecture critique de ce manuscrit.

\section{Summary}

\section{Cyclic nucleotide \\ phosphodiesterases}

Cyclic nucleotide phosphodiesterases (PDE) are classified into five families (PDE I to V) according to their primary sequence, their substrate specificities and their sensitivities to different modulators. PDE I, PDE II and PDE III can hydrolyze both cAMP and cGMP. Calmodulin stimulates PDE I activity. Cyclic GMP can itself modulate the hydrolysis of cAMP. It stimulates the hydrolysis of cAMP through PDE II, whereas it inhibits it through PDE III. The modulation of cAMP level by cGMP might explain either the synergistic or the opposite effects of cAMP and cGMP on some biological functions. PDE III are inhibited by some cardiotonic drugs (milrinone...) and are regulated in some tissues by a mechanism of phosphorylation. PDE IV selectively hydrolyze cAMP and are inhibited by rolipram. When both PDE III and PDE IV coexist in a cell type, PDE III might be involved in the regulation of basal level of cAMP whereas PDE IV might regulate higher level of cAMP. This might be due to the difference in the $K_{n}$ value of PDE III and PDE IV for cAMP. The last family, PDE V, specifically hydrolyzes cGMP and is involved in the regulation of smooth muscle relaxation and visual transduction. Selective PDE inhibitors are useful as pharmacological tools to increase cAMP or cGMP levels in target cells and, by this way, they are potential therapeutic agents for the treatment of clinical disorders such as heart failure, depression or asthma. 